\title{
VARIABILIDADE ESPACIAL DAS PERDAS E DA RESISTÊNCIA MECÂNICA DO SOLO À PENETRAÇÃO NO ARRANQUIO MECANIZADO DE AMENDOIM
}

\author{
Ariel Muncio Compagnon ${ }^{1}$, Rouverson Pereira da Silva², Marcelo Boamorte Raveli ${ }^{3}$, Diego Onofre Vidal ${ }^{4}$, Marcelo \\ Tufaile Cassia $^{5}$
}

\section{RESUMO}

A colheita mecanizada de amendoim é de fundamental importância no processo de produção da cultura, porém acarreta perdas consideráveis, principalmente na operação de arranquio. Assim, objetivou-se com este trabalho mensurar a variabilidade espacial das perdas e da resistência mecânica do solo à penetração (RP) na operação de arranquio mecanizado de amendoim em duas velocidades de arranquio. O trabalho foi realizado na região norte do estado de São Paulo. Os tratamentos avaliados foram constituídos por duas velocidades de deslocamento (V1=3,5 $\mathrm{km} \mathrm{h}^{-1}$ e V2=4,7 $\mathrm{km} \mathrm{h}^{-1}$ ) com dez repetições, com delineamento inteiramente casualizado (DIC). Após o arranquio, foram realizadas medições de RP utilizando-se um penetrômetro eletrônico, posicionado transversalmente às linhas de amendoim, a cada $0,01 \mathrm{~m}$ de profundidade, até a profundidade de $0,30 \mathrm{~m}$, e a cada 0,15 m de largura, até o total de 1,80 m. Nos mesmos pontos, foram determinadas as perdas no arranquio, classificadas como visíveis (PVA), invisíveis (PIA) e totais (PTA). A velocidade de deslocamento do conjunto trator-arrancador-invertedor não influenciou as perdas avaliadas e a RP. A mobilização de solo ocorreu até $0,13 \mathrm{~m}$ de profundidade, havendo desnivelamento transversal dos órgãos ativos do arrancador-invertedor. Houve correlação entre PVA e PIA, e entre PTA e RP, porém os coeficientes de determinação $\left(\mathrm{R}^{2}\right)$ situaram-se abaixo de $25 \%$.

Palavras-Chave: Arachis hypogaea L, maturação, mecanização

\section{ABSTRACT \\ SPATIAL VARIABILITY OF LOSSES AND SOIL MECHANICAL RESISTANCE TO PENETRATION IN MECHANIZED PEANUT DIGGING}

Mechanized peanut harvesting is of great importance in the crop production process but there are considerable losses, especially in the digging operation. The objective of this study was therefore to measure the spatial variability of peanut losses and soil mechanical resistance to penetration (RP) in the mechanized digging operation using two displacement speeds. The study was performed in the northern region of São Paulo State. Treatments consisted of two speeds $\left(\mathrm{V} 1=3.5 \mathrm{~km} \mathrm{~h}^{-1}\right.$ and $\left.\mathrm{V} 2=4.7 \mathrm{~km} \mathrm{~h}^{-1}\right)$ and ten replications using a completely randomized design (DIC). After the digging the RP measurements were obtained using an electronic penetrometer positioned across peanut lines, taking measurements at every $0.01 \mathrm{~m}$ of depth to a maximum of $0.30 \mathrm{~m}$ deep, and at every $0.15 \mathrm{~m}$ in width to a total of $1.80 \mathrm{~m}$. At the same points losses from digging were determined and classified as visible (PVA), invisible (PIA) and total losses (PTA). Speed of the tractor-digger did not influence the peanut losses evaluated or the RP. Soil mobilization occurred up to $0.13 \mathrm{~m}$ in depth and the level across the active parts of the digger was uneven. Correlations were observed between PVA and PIA, and between PTA and RP, but the determination coefficients $\left(\mathrm{R}^{2}\right)$ were less than $25 \%$.

Keywords: Arachis hypogaea L, maturation, mechanization

\section{Recebido para publicação em 21/09/2012. Aprovado em 24/05/2013.}

1 - Engenheiro Agrícola. Estudante de doutorado. Depto. de Engenharia Rural, FCAV/UNESP, arielcompagnon@gmail.com

2 - Engenheiro Agrícola, Professor Doutor, FCAV/UNESP/Jaboticabal-SP, rouverson@fcav.unesp.br

3 - Engenheiro Agrônomo, Estudante de doutorado, FCAV/UNESP/Jaboticabal-SP, m_boamorte@hotmail.com

4 - Engenheiro Agrônomo, Estudante de mestrado, FCAV/UNESP/Jaboticabal-SP, diego.vidal@cambuhy.com.br

5 - Engenheiro Agrônomo, Estudante de doutorado, FCAV/UNESP/Jaboticabal-SP, marcelocassia@gmail.com 


\section{INTRODUÇÃO}

A produção de amendoim no Brasil cresceu significativamente nos últimos anos, atingindo 296,7 mil toneladas na safra 2011/2012. O estado de São Paulo é responsável por $82 \%$ da produção nacional, destacando-se a região de Jaboticabal, responsável por $25 \%$ da produção estadual e $20 \%$ da brasileira (CONAB, 2012).

No sistema de colheita mecanizada o processo é iniciado com uma roçadora - para cultivares de porte ereto - seguindo-se as etapas de arranquio e enleiramento que são efetuadas simultaneamente com um único implemento, que corta as raízes, promove a vibração das plantas e realiza o enleiramento (NEVES, 2007). As leiras permanecem na lavoura para secagem e homogeneização natural e, então, outra máquina realiza o recolhimento do produto e separa os grãos do resto das plantas.

Apesar de as perdas na colheita representarem grandes prejuízos ao produtor, Sant'Ana et al. (2006) relatam que, para a colheita do amendoim, pouca importância é dada às pesquisas, tanto no que se refere ao momento adequado quanto à quantificação das perdas durante o arranquio. No entanto, segundo Olivatti et al. (2007), as perdas decorrentes do processo da colheita mecanizada do amendoim são inevitáveis, sendo necessário estabelecer condições de trabalho para minimizar estas perdas e garantir a viabilidade econômica da cultura.

Lamb et al. (2004) relataram que as perdas no arranquio são estimadas em $8 \%$ da produção total, mas quando o arranquio for realizado além do ponto ótimo de maturação podem chegar a $40 \%$. Rowland et al. (2006) encontraram perdas totais no arranquio da ordem de 35 a $50 \%$ da produtividade total em dois campos de produção na Geórgia (EUA). Os autores relatam que a avaliação da maturidade das vagens perdidas durante $\mathrm{o}$ arranquio mostrou claramente que a maioria das perdas ocorreu em vagens a partir da classe preta (mais maduras), representando de $25 \%$ a $50 \%$ de perdas na produção total.

Beam et al. (2002) afirmam que embora não esteja estabelecida na bibliografia, quando o amendoim é arrancado sob condições de solos menos férteis as perdas na colheita podem ser ainda maiores. Ainda com relação à questão dos solos, para Jordan et al. (2003), há suposições de que nos sistemas de preparo reduzido, as perdas possam ser maiores em relação aos sistemas de preparo convencional, devido à maior dificuldade no arranquio.

A resistência mecânica do solo à penetração (RP) é uma propriedade do solo adotada como indicativo da compactação, por apresentar forte relação com o crescimento radicular das plantas (PEDROTTI et al., 2001; BEUTLER et al., 2002), e por ser muito eficiente na identificação de estados de compactação, quando comparada à densidade do solo (FREDDI et al., 2006), além de ser de fácil e rápida determinação (MERCANTE et al., 2003). Fortemente influenciada pelo teor de água do solo, pode ser obtida por um penetrômetro ou penetrógrafo de cone (CAPPELLI et al., 2001). Para esse aparelho, a avaliação da resistência do solo à penetração pode ser realizada pelo índice de cone (IC), definido como a RP de uma ponta cônica, e expressa como a força por unidade de área da base do cone (unidade de pressão) (CUNHA et al., 2002).

Ribeiro (2009), ao compilar trabalhos sobre RP em Latossolo Vermelho Eutroférrico típico, estabeleceu a seguinte classificação para o IC: baixo (0 a $2 \mathrm{MPa}$ ); médio (2 a $4 \mathrm{MPa}$ ); alto (4 a 6 $\mathrm{MPa}$ ) e muito alto (acima de $6 \mathrm{MPa}$ ).

Diante do elevado nível de perdas encontradas na colheita do amendoim e da existência, no Brasil, de poucos trabalhos que abordem as possíveis causas dessas perdas, objetivou-se com este trabalho mensurar a variabilidade espacial das perdas e da resistência mecânica do solo à penetração na operação de arranquio mecanizado de amendoim em duas velocidades de arranquio.

\section{MATERIAL E MÉTODOS}

O experimento foi realizado na Fazenda Santa Maria, na região norte do estado de São Paulo, localizada nas coordenadas geodésicas: latitude $21^{\circ} 14^{\prime} 58^{\prime \prime} \mathrm{S}$ e longitude $48^{\circ} 24^{\prime} 18^{\prime \prime} \mathrm{W}$, com altitude média de $635 \mathrm{~m}$. O clima da regiãoé Aw (subtropical), de acordo com a classificação de Köeppen (1948). A determinação da textura do solo foi obtida por meio de quatro amostras, retiradas aleatoriamente da área experimental, as quais foram misturadas formando 
uma amostra homogênea, que posteriormente foi enviada para o Laboratório de Solos da UNESP/ Jaboticabal para a determinação das proporções de argila, silte e areia (Quadro 1).

O trator utilizado foi um Valtra BM 120, com potência de 88,2 kW (120 cv), nas marchas de trabalho $3^{\mathrm{a}} \mathrm{L}$ Baixa à $1500 \mathrm{rpm}$ (V1), e $3^{\mathrm{a}}$ L Alta, à $1500 \mathrm{rpm}$ (V2), tracionando um arrancadorinvertedor montado 2x1 (2 linhas x 1 leira), marca Santal modelo AIA-BM2, possuindo largura de trabalho de 1,80 m (Figura 1).

A área de amendoim foi semeada em 10 de novembro de 2010, com a variedade IAC Runner 886, com espaçamento entrelinhas de $0,90 \mathrm{~m}$ e o arranquio foi realizado 140 dias após a semeadura.

Os tratamentos avaliados foram constituídos por duas velocidades de deslocamento (V1 = 3,5 $\mathrm{km} \mathrm{h}^{-1}$ e $\mathrm{V} 2=4,7 \mathrm{~km} \mathrm{~h}^{-1}$ ) com dez repetições, totalizando vinte amostras, em um delineamento experimental inteiramente casualizado (DIC).

Após o arranquio, foram realizadas medições de resistência mecânica do solo à penetração (RP) utilizando-se o penetrômetro eletrônico DLG, modelo PNT-TITAN, que segue a norma ASAE S313.2 (ASAE, 1998). Os dados foram coletados transversalmente às linhas de amendoim, a cada 0,01 $\mathrm{m}$ de profundidade, até a profundidade de $0,30 \mathrm{~m}$, e a cada $0,15 \mathrm{~m}$ de largura, até o total de 1,80 m (duas linhas de arranquio). Os pontos foram espaçados em $20 \mathrm{~m}$, em um total de 4 leiras (5 pontos por leira), sendo as duas primeiras correspondentes ao tratamento $\mathrm{V} 1$ e as duas subsequentes ao tratamento $\mathrm{V} 2$, totalizando 20 pontos (10 por tratamento). O sentido de deslocamento do conjunto tratorarrancador foi mantido sempre o mesmo, para evitar possíveis erros devido à configuração do arrancador-invertedor e do terreno.

Para avaliação das perdas foi utilizada uma armação metálica de área de $2 \mathrm{~m}^{2}$ (1,80 m x 1,11 $\mathrm{m})$, nos mesmos 20 pontos da análise de RP, sendo realizada as seguintes análises:

- Perdas Visíveis no Arranquio (PVA): correspondem às vagens e grãos de amendoim encontradas sobre a superfície do solo após a operação de arranquio.

- Perdas Invisíveis no Arranquio (PIA): compreende as vagens e grãos de amendoim encontradas abaixo da superfície do solo.

- Perdas Totais do Arranquio (PTA): correspondem à soma das perdas visíveis e invisíveis no arranquio.

Em relação à qualidade da operação de arranquio foram retiradas 4 amostras para determinação da produtividade da cultura, a qual foram arrancadas manualmente todas as vagens das plantas de amendoim contidas na área da armação

Quadro 1. Análise granulométrica do solo da área de estudo

\begin{tabular}{ccccc}
\hline Argila & Limo & Areia Fina & Areia Grossa & Classe Textural \\
- & - & & \\
\hline 176 & 28 & 435 & 361 & Média \\
\hline
\end{tabular}

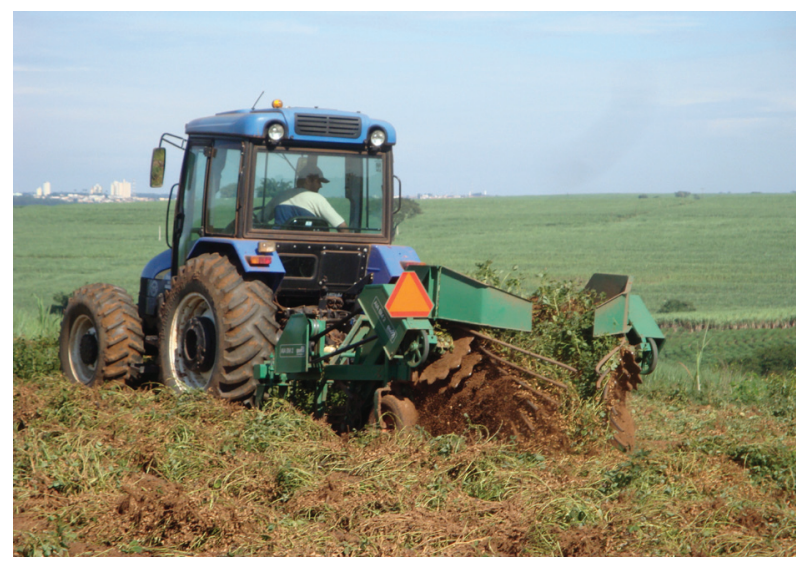

(a)

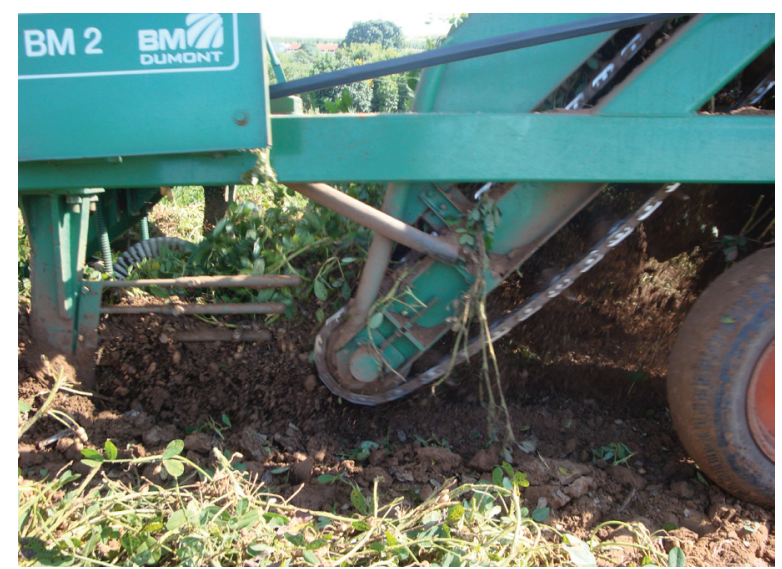

(b)

Figura 1. Conjunto trator-arrancador-invertedor utilizado (a). Vista lateral do arrancador-invertedor. 
de $2 \mathrm{~m}^{2}$ (SILVA; MAHL, 2008) e 20 amostras de 100 vagens por amostra para determinação da maturação, pelo método "Hull Scrape" (WILLIAMS; DREXLER, 1981). Foram coletadas 4 amostras do solo utilizando-se um trado holandês nas camadas de 0,00-010; 0,10-0,20 e 0,20-0,30 m de profundidade, sendo estas acondicionadas em recipientes de alumínio para determinação do teor de água, conforme metodologia recomendada por EMBRAPA (1997).

Os dados de PVA, PIA e PTA foram submetidos à análise de variância pelo teste $\mathrm{F}$, sendo analisados pelo programa estatístico SISVAR e quando houve significância das médias, aplicou-se o teste de Tukey a $5 \%$ de probabilidade. Procedeuse a montagem dos gráficos de variabilidade espacial da RP e da matriz de correlação entre as perdas, maturação das vagens e a RP por meio do programa Minitab ${ }^{\circledR}$, selecionando-se por meio do coeficiente de correlação de Pearson e do valor-P de probabilidade $(\mathrm{P}<0,05)$ as combinações de maior correlação linear.

\section{RESULTADOS E DISCUSSÃO}

A área avaliada apresentou produtividade média de $5.728 \mathrm{~kg} \mathrm{ha}^{-1}$, teor de água das vagens de $65,3 \%$ e maturação de $85,6 \%$. Com relação ao solo, o teor de água nas camadas de 0,00-0,10;0,10-0,20 e 0,20-0,30 $\mathrm{m}$ foram, respectivamente, 11,$06 ; 11,23$ e $11,31 \%$, demonstrando a homogeneidade do teor de água nas camadas avaliadas.

Não houve efeito da variação da velocidade para as perdas visíveis, invisíveis e totais no arranquio (Quadro 2). Os coeficientes de variação foram 35,$04 ; 41,13$ e $33,18 \%$ para PVA, PIA e PTA, respectivamente, valores estes considerados muito altos. Valores de até 30\% são bons indicativos da coleta de dados em trabalhos de campo (PIMENTEL-GOMES; GARCIA, 2002). Porém, em estudos de perdas na colheita de amendoim, tem sido encontrado altos valores dos coeficientes de variação (BARROZO et al., 2008), assim como em outras culturas (CAMPOS et al., 2005; MESQUITA, 2001), justificando que esses altos valores refletem a grande variabilidade das perdas em função do local amostrado e da produção nas parcelas.

Analisando a percentagem das perdas em relação à produtividade, observou-se que a média encontrada de PVA foi de $4,8 \%$, e PIA, de $4,9 \%$. Gameiro et al. (2008) diagnosticaram as perdas no arranquio mecanizado de amendoim cultivar IAC Runner 886, com o arrancador-invertedor tracionado à velocidade média de $5,5 \mathrm{~km} \mathrm{~h}^{-1}$, e encontraram valores de 5 e $13 \%$ para PVA e PIA, respectivamente. Com relação à percentagem de PTA em relação à produtividade, a média encontrada no presente trabalho foi de $9,74 \%$, corroborando com Mahl et al. (2008), que encontraram perdas de

Quadro 2. Síntese da análise de variância e teste de médias de perdas visíveis (PVA), invisíveis (PIA) e totais (PTA) no arranquio para as velocidades de arranquio testadas $\left(\mathrm{km} \mathrm{h}^{-1}\right)$

\begin{tabular}{lccc} 
& \multicolumn{3}{c}{ Perdas $\left(\mathrm{kg} \mathrm{ha}^{-1}\right)$} \\
\cline { 2 - 4 } & PVA & PIA & PTA \\
\hline Velocidade $(\mathrm{V})$ & & & \\
3,5 & $277,79 \mathrm{a}$ & $289,91 \mathrm{a}$ & $567,70 \mathrm{a}$ \\
4,7 & $276,76 \mathrm{a}$ & $271,86 \mathrm{a}$ & $548,63 \mathrm{a}$ \\
\hline Probabilidade $\mathrm{P}<0,05$ & $0,9814^{\mathrm{ns}}$ & $0,7310^{\mathrm{ns}}$ & $0,8205^{\mathrm{ns}}$ \\
\hline CV $(\%)$ & 35,04 & 41,13 & 33,18 \\
\hline
\end{tabular}

$\overline{\text { Em cada coluna, para cada fator, médias seguidas de mesmas letras não diferem entre si, pelo teste de Tukey a } 5 \% \text { de }}$ probabilidade. CV: coeficiente de variação. ${ }^{\text {ns: }}$ Não significativo a $5 \%$ de probabilidade, pelo teste de $\mathrm{F}$. 
$9,2 \%$ na avaliação do arranquio na região noroeste do Paraná.

A velocidade de arranquio não influenciou na profundidade de trabalho do arrancador-invertedor (Figura 2), pois houve mobilização do solo, até próximo a $0,13 \mathrm{~m}$ de profundidade, fato observado pelos valores de RP na mesma profundidade, o que pode ser explicado pelo fato de as velocidades estarem dentro da faixa recomendada para o arranquio de amendoim (3 a $\left.5 \mathrm{~km} \mathrm{~h}^{-1}\right)$.

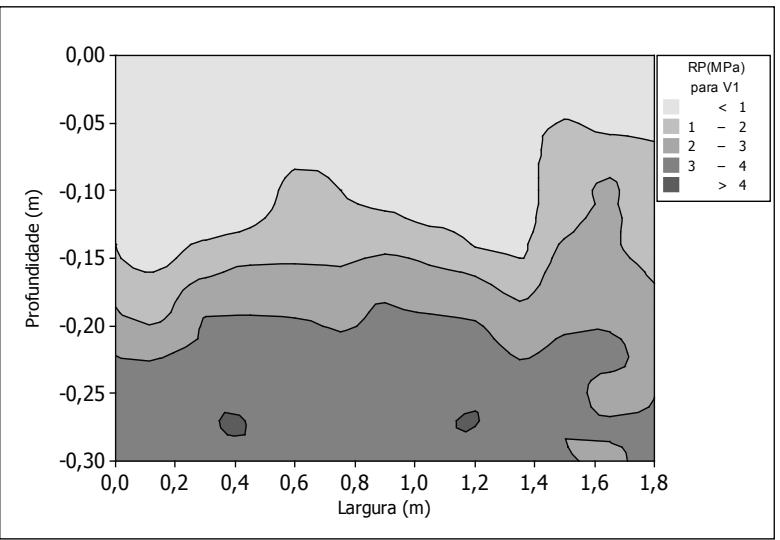

(a)

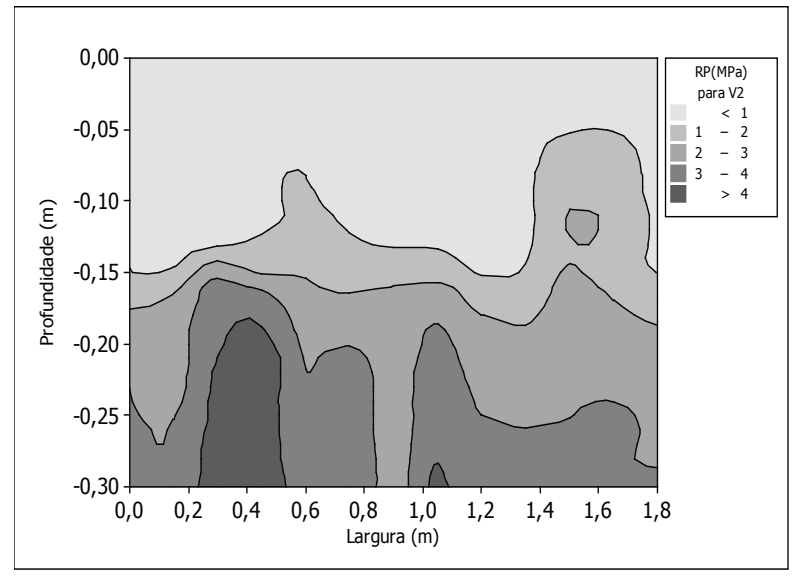

(b)

Figura 2. Resistência mecânica do solo à penetração (MPa) após o arranquio, nas velocidades de 3,5 (a) e $4,7 \mathrm{~km} \mathrm{~h}^{-1}$ (b).
A partir da profundidade de 0,13 m, não há mais influência do arrancador-invertedor na mobilização do solo, pois a RP aumenta. Segundo Ribeiro (2009), valores abaixo de $2 \mathrm{MPa}$, observados até 0,16 m, são considerados baixos, evidenciando então a ação do arrancador-invertedor. A partir de $0,19 \mathrm{~m}$, nota-se que a RP atinge valores próximos ou superiores a $3 \mathrm{MPa}$, o que, segundo Ribeiro (2009), são considerados médios, indicando que não houve revolvimento pelos implementos de preparo do solo para a semeadura do amendoim.

Porém, ao se analisar o perfil da RP, observase que o arrancador-invertedor encontrava-se com seus órgãos ativos desnivelados transversalmente, visto que no lado direito dos perfis, há maior RP quando comparado com o lado esquerdo, em relação às camadas de profundidade de 0,07 a $0,15 \mathrm{~m}$, indicando que a faca do lado direito trabalhou com a metade da profundidade da faca da esquerda. Uma vez que a regulagem dos braços inferior direito e esquerdo do sistema hidráulico de três pontos do trator foi devidamente controlada, as hipóteses para este desnivelamento restringiramse à existência de algum problema estrutural na montagem da faca direita do arrancador.

Os coeficientes de correlação (Quadro 3) demonstram existir correlação entre PVA e PIA, PTA e RP e de PVA e PIA com PTA. As correlações de PVA e PIA com PTA referem-se ao fato de que as perdas totais são dependentes dos valores de PVA e PIA.Apesar da existência de correlação significativa, verificou-se por meio da análise de regressão que os valores dos coeficientes de determinação $\left(\mathrm{R}^{2}\right)$ situaram-se abaixo de $25 \%$ (Figuras 3 e 4 ). Isto pode ser explicado pela grande variabilidade dos valores encontrados nas avaliações de perdas, indicados pelos altos valores dos coeficientes de variação observados (Quadro 2), corroborando com Barrozo et al. (2008). Para as demais variáveis não foram verificadas a existência de correlação.

Quadro 3. Matriz dos coeficientes de correlações de Pearson para as variáveis analisadas

\begin{tabular}{lcccc}
\hline Variáveis & PVA & PIA & PTA & MAT \\
\hline PIA & $0,512^{*}$ & - & & - \\
PTA & $0,844^{*}$ & $0,893^{*}$ & - & - \\
MAT & $0,304^{\text {ns }}$ & $0,190^{\text {ns }}$ & $0,278^{\text {ns }}$ & - \\
RP & $-0,407^{\text {ns }}$ & $-0,423^{\text {ns }}$ & $-0,477^{*}$ & $-0,362^{\text {ns }}$ \\
\hline
\end{tabular}

PVA: perdas visíveis no arranquio. PIA: perdas invisíveis no arranquio. PTA: perdas totais no arranquio. MAT: maturação das vagens. RP: resistência mecânica do solo à penetração. ${ }^{\text {ns: }}$ Não significativo; * : Significativo a 5\% de probabilidade. 


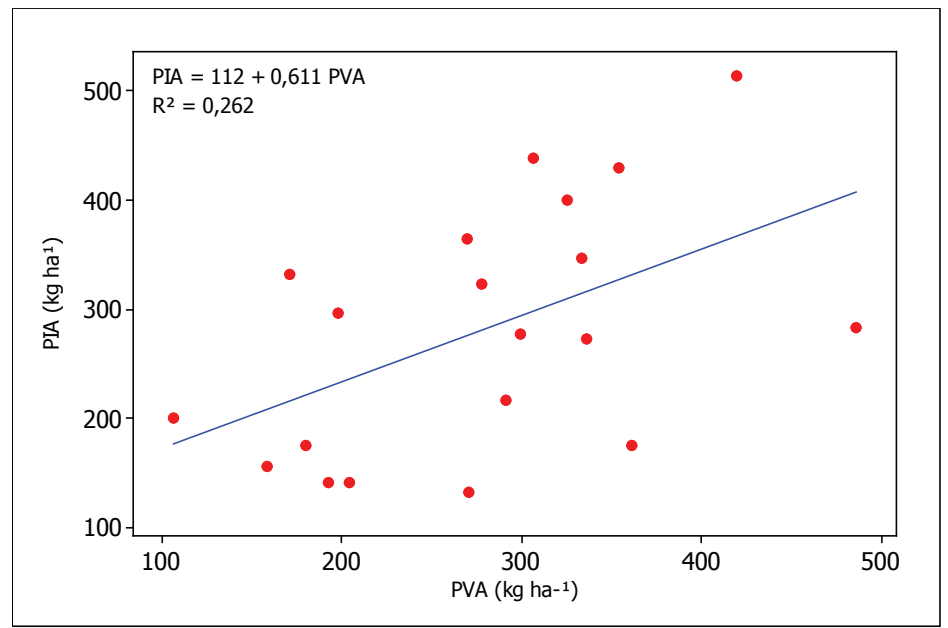

Figura 3. Regressão linear para PIA x PVA.

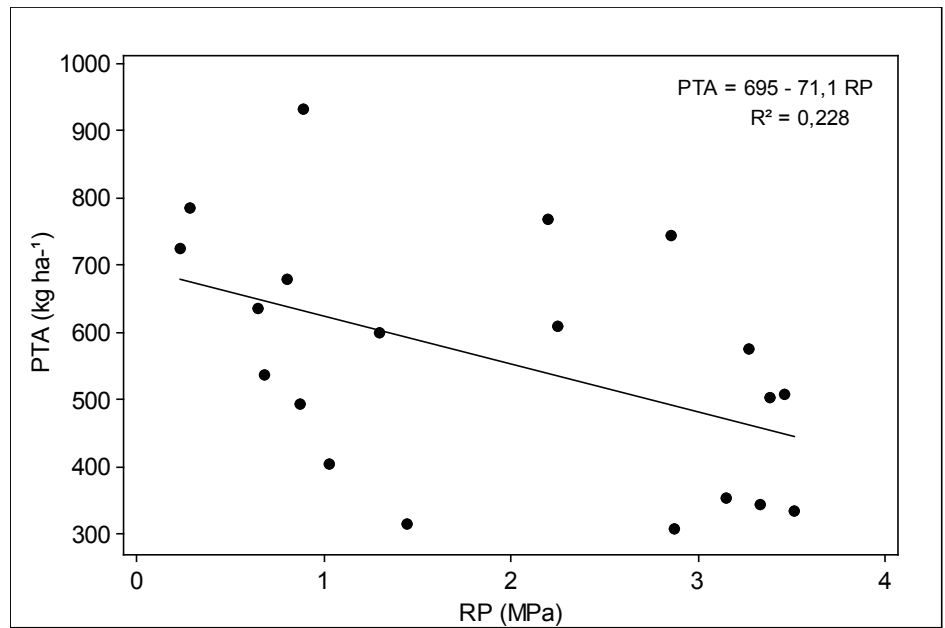

Figura 4. Regressão linear para RP x PTA.

\section{CONCLUSÕES}

- A velocidade de deslocamento do conjunto trator-arrancador-invertedor não influenciou as perdas visíveis, invisíveis e totais no arranquio, assim como para a resistência mecânica do solo à penetração.

- Houve correlação entre perdas visíveis e invisíveis no arranquio e entre resistência mecânica do solo à penetração e perdas totais no arranquio, porém o coeficiente de determinação $\left(\mathrm{R}^{2}\right)$ foi baixo.

- Não houve correlação para a maturação das vagens e as perdas visíveis, invisíveis e totais no arranquio.

\section{REFERÊNCIAS BIBLIOGRÁFICAS}

ASAE. Soil cone penetrometer: ASAE standard S313.2, St. Joseph. ASAE. p.820-821. 1988.

BARROZO, L.M.; SILVA, R.P.; SADER, R.; SIMÕES, R.R. Perdas na colheita mecanizada do amendoim (Arachis hypogaea L.) em função da velocidade de arranquio. In: ENCONTRO SOBRE A CULTURA DO AMENDOIM, 2000, Jaboticabal. Anais... Jaboticabal: FUNEP. CD-ROM.

BEAM, J.B.; JORDAN, D.L.; YORK, A.C.; ISLEIB, T.G.; BAILEY, J.E.; MCKEMIE, T.E.; SPEARS, J.F.; JOHNSON, P.D. Influence of prohexadione calcium on pod yield and pod loss of peanut. Agronomy Journal, Madison, v.94, p.331-336, 2002.

BEUTLER, N.A.; CENTURION, J.F.; SOUZA, Z.M.; SILVA, L.M. Utilização dos penetrômetros de impacto e de anel dinamométrico em latossolos. Engenharia Agrícola, Jaboticabal, v.22, p.191-199, 2002.

CAMPOS, M.A.O.; SILVA, R.P.; MESQUITA, H.C.B.; ZABANI, S. Perdas na colheita mecanizada de soja no estado de Minas Gerais. Engenharia Agrícola, Jaboticabal, v.25, p.207-213, 2005.

CAPPELLI, N.L.; UMEZU, C.K.; CAMPOS, R.F. Push cone eletrônico de baixo custo para estudo da resistência mecânica ao desenvolvimento radicular das plantas. Revista Brasileira de Engenharia Agrícola e 
Ambiental, Campina Grande, v.5, p.525-529, 2001.

CONAB. Acompanhamento da safra brasileira: grãos, safra 2011/2012, oitavo levantamento: maio/2012. 2012. Disponível em: <http://www.conab.gov.br/OlalaCMS/ uploads/arquivos/12 $05 \quad 10 \quad 08 \quad 49 \quad 52$ boletim maio 2012.pdf $>$. Acesso em: 05 de jun. 2012.

CUNHA, J.P.A.R.; VIEIRA, L.B.; MAGALHÃES, A.C. Resistência mecânica do solo à penetração sob diferentes densidades e teores de água. Engenharia na Agricultura, Viçosa, v.10, p.1-7, 2002.

EMBRAPA. Serviço Nacional de Levantamento e Conservação do Solo. Manual de métodos e análise de solo. Rio de Janeiro, 1997. 212p.

FREDDI, O.S.; CARVALHO, M.P.; VERONESI JÚNIOR, V.; CARVALHO, G.J. Produtividade do milho relacionada com a resistência mecânica à penetração do solo sob preparo convencional. Engenharia Agrícola, Jaboticabal, n.26, p.113-121, 2006 .

GAMEIRO, M.C.; MAHL, D.; SILVA, M.B.; LOPES, U.M.; WICHMANN, V.A. Avaliação de perdas visíveis e invisíveis no arranquio mecanizado do amendoim na região Noroeste do Paraná. In: ENCONTRO SOBRE A CULTURA DO AMENDOIM, 2008, Jaboticabal. Anais... Jaboticabal: FUNEP. CD-ROM.

JORDAN, D.L.; BARNES，J.S.; BOGLE，C.R.; BRANDENBURG, R.L.; BAILEY, J.E.; JOHNSON, P.D.; CULPEPPER, A.S. Peanut response to cultivar selection, digging date, and tillage intensity. Agronomy Journal, Madison, v.95, p.380-385, 2003.

KÖEPPEN, W. Climatologia: con um estúdio de los climas de la Tierra. México: Fondo de Cultura Economica, 1948. 478p.

LAMB, M.C.; MASTERS, M.H.; ROWLAND, D.; SORENSEN, R.B.; ZHU, H.; BLANKENSHIP, P.D.; BUTTS, C.L. Impact of sprinkler irrigation amount and rotation on peanut yield. Peanut Science, Raleigh, v.31, p.108-113, 2004.

MAHL, D.; WESSLER, V.; OLIVATTI, B.M. Losses during the process of mechanized harvesting of peanuts obtained with two harvesters. In: INTERNATIONAL CONFERENCE OF AGRICULTURAL ENGINEERING, 2008, Foz do Iguaçu: SBEA. Anais... CD-ROM. p.1-4.

MERCANTE, E.; URIBE-OPAZO, M.A.; SOUZA, E.G. Variabilidade espacial e temporal da resistência mecânica do solo à penetração em áreas com e sem manejo químico localizado. Revista Brasileira de Ciência do Solo, Viçosa, n.27, p.1149-1159, 2003.

MESQUITA, C.M. Caracterização da colheita mecanizada da soja no Paraná. Engenharia Agrícola, Jaboticabal, v.21, p.197-205, 2001.

NEVES, I.N. Dossiê Técnico: Cultivo do Amendoim, Rede de Tecnologia da Bahia - RETEC/BA, 2007, 20p.

OLIVATTI, B.M.; MAHL, D.; WESSLER, V. Perdas no arranquio mecanizado do amendoim em função da configuração do arrancador-invertedor e velocidade de deslocamento. In: IV ENCONTRO DO AMENDOIM, 2007, Jaboticabal: FUNEP. Anais... CD-ROM.

PEDROTTI, A.; PAULETTO, E.A.; CRESTANA, S.; FERREIRA, M.M.; DIAS JUNIOR, M.S.; GOMES, A.S.; TURATTI, A.L. Resistência mecânica à penetração de um planossolo submetido a diferentes sistemas de cultivo. Revista Brasileira de Ciência do Solo, Viçosa, n.25, p.521-529, 2001.

PIMENTEL-GOMES, F.; GARCIA, C.H. Estatística aplicada a experimentos agronômicos e florestais: exposição com exemplos e orientações para uso de aplicativos. Piracicaba: FEALQ, 2002. 309p.

RIBEIRO, C.A. Variabilidade espacial da resistência mecânica do solo à penetração em áreas mecanizadas em função do número de cortes da cana-de-açúcar (Saccharum officinarum). 2009. 69p. Dissertação (Mestrado em Ciência do Solo) - Faculdade de Ciências Agrárias e Veterinárias, Universidade Estadual Paulista, Jaboticabal, São Paulo, 2009.

ROWLAND, D.L.; SORENSEN, R.B.; BUTTS, C.L.; FAIRCLOTH, W.H. Determination of maturity and degree day indices and their success in predicting peanut maturity. Peanut Science, Raleigh, n.33, p.125-136, 2006.

SANT'ANA, C.; SILVA, R.P.; GARRAFONI, N.C.; CORTEZ, J.W.; FURLANI, C.E.A. Levantamento das perdas quantitativas na colheita mecanizada de amendoim na região de Guatapará - SP. In: CONGRESSO BRASILEIRO DE ENGENHARIA AGRÍCOLA, 2006, João Pessoa: SBEA, 2000. Anais... CD-ROM. p.1-4.

SILVA, R.P.; MAHL, D. Determinação de perdas na colheita de amendoim. Jaboticabal. 2008, 3p. Folder.

WILLIAMS, E.J.; DREXLER, J.S. A non destructive method for determining peanut pod maturity. Peanut Science, Raleigh, n.8, p.134-141, 1981. 